\title{
Using a menstrual emotional stroop task test (MEST) to measure emotional responses to menstrual cycle pain
}

\begin{abstract}
The purpose of this study was to configure and test a Menstrual-related Emotional Stroop Task (MEST) to assess differences in emotional responsiveness of healthy cycling women in order to identify the effects of pain anticipation, anxiety and depression symptomatology. Participants included 40 healthy women between 21 and 51years of age, with regular menstrual cycles who completed questionnaires on socio-demographics, menstrual cycle characteristics, nicotine use, and alcohol consumption. The numeric pain rating scale (Visual Analogue Scale or VAS) was used to assess perceived levels of menstrual pain. Twice during their menstrual cycle (follicular phase and premenstrual phase, 3-5days before menses), we administered the MEST, the VAS, the Beck Anxiety Inventory (BAI) and the Beck Depression Inventory II (BDI-II). Subjects reporting higher levels of pain (VAS score $>7, n=7$ ) had the longest reaction time in the MEST, specifically in the premenstrual phase compared to those with mild (VAS score $1-3, n=22$ ) levels of menstrual pain, possibly indicating an "anticipatory stress" emotional response to their impending menses. Linear regression analysis revealed correlations between depression levels and performance in MEST during the premenstrual phase. Overall, our data suggest that emotional responses are deeply affected by the anticipation of menstrual pain and are associated with depression. Moreover, this study indicates that MEST is an effective tool to obtain information about the emotional impact of menstrual pain and identifies associated impairments in attentional processes. These results have implications for the development of pain management skills and psycho-education programs for women suffering from painful conditions such as endometriosis.
\end{abstract}

Keywords: pelvic pain, menstrual cycle, depression, anxiety, emotional stroop task
Volume 3 Issue 4 - 2017

\author{
Mélanis Rivera Rodríguez,' Idhaliz Flores, ${ }^{2}$ \\ Summer F Acevedo 3 \\ 'Psychology Program, Ponce Health Sciences University, USA \\ ${ }^{2}$ Department of Microbiology and Ob-Gyn, Ponce Health \\ Sciences University, USA \\ ${ }^{3}$ Department of Psychiatry, UT South western Medical Center, \\ USA
}

Correspondence: Summer F Acevedo, PhD Instructor Department of Psychiatry, UT Southwestern Medical Center, Dallas, TX 75235, USA, Tel (24I) 648II74,

Email Summer.Acevedo@UTSouthwestern.edu

Received: May 25, 2016 | Published: April 07, 2017
Abbreviations: EST, emotional stroop task; MEST, menstrual emotional stroop task; PMS, premenstrual syndrome; PMDD, premenstrual dysphoric disorder; PTSD, post traumatic stress disorder; VAS, the visual analogue scale; BAI, beck anxiety inventory; BDI-II, beck depression inventory

\section{Introduction}

The menstrual cycle is divided in different phases based on cyclic levels of the ovarian sex steroids estradiol and progesterone. Generally, the menstrual cycle is not associated with symptomatology and is regarded as a natural body process in females. However, in some cases menses comes accompanied by symptoms that include pelvic pain and psychological changes, alone or in combination, which often impact women mentally, physically and socially interfering with daily living. ${ }^{2-4}$ The etiology of menstrual-related pelvic pain is not easily discernible. There are many disorders of the reproductive tract, gastrointestinal, urological organs, musculoskeletal and neuropsychological systems that may be associated with pelvic pain in women. ${ }^{5}$ It is important to evaluate the causes of pain in each patient and other factors impacting the individual experience of pain, such as thresholds, mental health status or stress among others, in order to provide more effective targeted treatments.

Pain is the most common reason patients seek primary care treatment, accounting for $40 \%$ of all problem-focused outpatient visits. ${ }^{6}$ If pain is not managed over time it can become chronic and difficult to endure leading to conditions such as Post-Traumatic Stress Disorder (PTSD). ${ }^{7}$ Moreover, pain is a complex function, depending on cognition, emotions, context, and biological status of neuronal structures. ${ }^{8}$ Pain has much broader implications for health as an integrated framework compared to mood and behavioral syndromes alone. ${ }^{9}{ }^{10}$ Presently, there is a lack of research on cyclic menstrual pain and the emotional responses associated with it. ${ }^{11}$ As a result, there is limited understanding of the mechanisms underlying pain associated with the menstrual cycle, leading to a lack of effective treatments and approaches to minimize its impact.

In addition to the pain associated with menstruation, as many as $3-5 \%$ of women currently suffer from the luteal mood and anxiety changes that are characteristic of premenstrual dysphoric disorder (PMDD). ${ }^{12}$ Unlike healthy women who display differences in left amygdala reactivity between menstrual phases, ${ }^{12,13}$ women with PMDD have attenuated bilateral amygdala reactivity in the follicular phase, high trait anxiety and increased right amygdala reactivity during their luteal phase compared to follicular. ${ }^{12}$ Serum progesterone levels correlate to amygdala activity and emotional responsiveness during different phases of the menstrual cycle in healthy women; ${ }^{14,15}$ thus measuring emotional interference of tasks that target amygdala functioning may be useful in understanding more about menstrual related changes in mood and anxiety behaviors. ${ }^{16}$ 
The Emotional Stroop Task (EST) was designed as a tool to measure emotional interference by examining the association of emotional responses to a given situation or health issue. During the EST the subject is presented color words in a congruent (e.g., the word blue written in blue ink) or incongruent (e.g., the word blue written in yellow ink) format. ${ }^{17}$ When instructed to ignore the word and indicate the color, subjects are usually slower to name incongruent stimuli in comparison with congruent stimuli. This effect is termed interference or the Stroop Effect. ${ }^{18,19}$ Studies suggest that subjects tend to name items that are associated with emotions at slower rate, because they may be more distinctive than neutral ones. ${ }^{20,21}$ For instance, subjects with disorders like anxiety, depression and/or eating disorders, are generally slower to name words associated with their disorder. ${ }^{22-24}$ Therefore, we hypothesized that women with menstrual pain will be slower to name relevant words associated with the menstrual cycle and symptoms related to their period. In these women, we speculated, the processing of negative or threatening word content will interfere with attention allocation to the more superficial task of labeling word color, resulting in longer EST response latencies. ${ }^{25}$

The objectives of this study were to configure a menstrual phasespecific Emotional Stroop Task (MEST) to measure the emotional responses and reaction time to menses-related words, The MEST was administered to women reporting different levels of cyclic menstrual pain during two phases of their menstrual cycles. To our knowledge, there is only one other small study that has used a similar approach (an EST that presented menstrual related words paired with faces) to explore if women with and without premenstrual syndrome (PMS) would respond differently. ${ }^{26}$ The present study was designed to help fill this gap in the current knowledge by developing and testing the MEST, a unique, easy to apply tool to examine the association of emotional responses and cyclic menstrual pain.

\section{Materials and methods}

\section{Participants}

Subjects in this study were females between the ages of 21 and 51 , with a regular 28 to 35 days menstrual cycle, who agreed to participate two times at different phases of their menstrual cycle within a threemonth period. Subjects were chosen upon availability from those who responded to invitations to participate in the study through flyers and promotional emails. Exclusion criteria included women with endometriosis or medically diagnosed dysmenorrhea, current oral contraception pill, irregular menstrual cycle (shorter than 28days or longer than 35days), history of mental impairments or psychiatric conditions, and history of neuropathological and/or neuropsychological conditions. Additional exclusion criteria included history of substance abuse that may interfere with attention, lack of concentration necessary for psychological testing, being colorblind or having other sensory limitations that may cause interference with the computerized testing. Efforts were in place to recruit subjects with similar age range, education levels, and health status. Power analysis indicated that a sample size of 40 women was sufficient to detect significant differences between the groups. Based on published EST, to detect a $10 \%$ difference $(0.50 \mathrm{SD}, \mathrm{p}<0.05)$ with $85 \%$ power in EST responses within healthy subjects we recruited a minimum of 20 subjects per group with two groups of subjects randomly counterbalanced on which was their first session. ${ }^{27}$ To conduct the investigation, approximately 40 participants were screened confidentially using an IRB approved socio-demographic questionnaire. All participants received a small compensation (five dollars) for their time upon test completion.

\section{Study design}

The research design for this study was a within-subjects design. Study subjects and the investigator met at two set times of their individual's menstrual cycle. The first session was scheduled 7-10days after the onset of menses (follicular) and the second session was scheduled 4-5days prior to the expected start of their menstrual period (luteal or pre-menstrual). At those pre-determined appointments the subjects completed questionnaires and were administered the MEST. Women were divided into two groups and administered the MEST. To control for order effects, the session order for each of the groups was counterbalanced by commencing the assessment of the two study groups in different cycle phases ${ }^{28}$ with one group having their first session during the follicular phase and the other during the luteal phase. The possible drawbacks of repeated measures are the differential carryover effects as a result of practice or exposure effects, which were managed by randomizing the order and color of word presentation in the computerized version of the MEST. No differences were found based on which was the first session so data was combined.

\section{Study questionnaires}

During the first session, a 5 minute questionnaire was administered to assess the women's socio demographic and gynecologic history information to obtain a basic profile of the participants. During the first and second sessions, the BDI-II was completed to evaluate depression indicators and the BAI was administered to assess anxiety symptoms. Levels of menstrual cyclic pain were also assessed during the two sessions using the self-reported VAS pain scale.

The visual analogue scale (VAS): This scale is a common form of response option in health outcomes studies, often used to measure levels of pain. It is generally presented as a single line of $100 \mathrm{~mm}$ with anchor words at either end (e.g., no pain, worst possible pain). For the study, the VAS was incorporated with the Numeric Rating Scale (NRS) to offer the participants a range of numerical, increasing values to describe their pain, thus providing an accurate report of the perceived pain during their menstrual period.

Beck anxiety inventory (BAI): This questionnaire is a 5 to 10 minutes inventory developed to address the need for an instrument that would reliably discriminate anxiety from depression while displaying convergent validity. ${ }^{29}$ The respondent is asked to rate how much he or she has been bothered by each symptom over the past week on a 4-point scale ranging from 0 to 3 . The items are summed to obtain a total score that can range from 0 to 63 . Scores are divided into categories including: minimal (0-7 points), mild (8-15 points), moderate (16-25 points) or severe (26-63 points).

Beck depression inventory, second edition (BDI-II): This questionnaire is a 5 to 10 minutes self-administered or verbal inventory. ${ }^{30}$ The BDI-II consists of 21 items to assess the intensity of depression symptoms in clinical and healthy patients. Each item is composed of a list of four statements arranged in increasing severity about a particular symptom of depression. These new items bring the BDI-II into alignment with DSM-IV, TR criteria. In this instrument, similar to the BAI, the items are summed to obtain a total score that can range from 0 to 63 . Scores are divided into four categories: minimal ( $0-13$ points), mild (14-19 points), moderate (20-28 points) or severe (29-63 points).

Menstrual-related emotional stroop task (MEST): This task is a modified version of the California Standardized Test Color Stroop Task. ${ }^{31}$ A Spanish translation was used in our study population. 
In this task, participants were asked to press the button with the corresponding color (e.g., red, green, yellow \& blue) in which a series of consecutively presented words were presented in the computer screen as fast as they could. Two types of words were used in the MEST: menstrual cycle related threatening words and neutral words. The 45 menstrual-related words reflected symptoms of painful gynecological conditions (e.g., blood, cramps, nausea, agony, misery, etc.). The 45 neutral words were household furnishings or objects (e.g., paint, walk, chair, file, cup, cable, table, refrigerator, lamp, desk, curtain, etc) (Table 1). A single- item variant MEST words were then presented on a computer monitor for 1.5 second duration with 0.5 second inter-stimulus intervals.

Table I Words used to configure menstrual related emotional stroop task (MEST)

\begin{tabular}{|c|c|c|c|c|c|c|c|}
\hline \multicolumn{4}{|l|}{ Spanish } & \multicolumn{4}{|l|}{ English } \\
\hline Threatening & & Neutral & & Threatening & & Neutral & \\
\hline Nauseas & Estrógeno & Gorro & Teclado & Nausea & Estrogen & Cap & Keyboard \\
\hline Hinchazón & Miseria & Carta & Asfalto & Swelling & Sick & Letter & Asphalt \\
\hline Dolor & Enferma & Abrir & Próximo & Pain & Misery & Open & Next \\
\hline Penetración & Tampones & Pájaro & Mensaje & Penetration & Tampons & Bird & Message \\
\hline Menstruación & Impotencia & Espejo & Lavadora & Menstruation & Helplessness & Mirror & Machine \\
\hline Calambres & Rechazo & Toalla & Acueducto & Cramps & Rejection & Towel & Aqueduct \\
\hline Sangre & Inflamación & Puerta & Vaso & Blood & Inflammation & Door & Cup \\
\hline Insomnio & Fibrosis & Líquido & Cable & Insomnia & Fibrosis & Liquid & Cable \\
\hline Cansancio & Estreñimiento & Piscina & Buzón & Tiredness & Constipation & Pool & Mailbox \\
\hline Infertilidad & Tumor & Montaña & Andar & Infertility & Tumor & Mountain & Walk \\
\hline Fatiga & Ansiedad & Cemento & Cerrar & Fatigue & Anxiety & Cement & Close \\
\hline Maligno & Tejido & Algodón & Portón & Malignant & Tissue & Cotton & Fence \\
\hline Útero & Debilidad & Escalera & Nevera & Uterus & Debility & Ladder & Refrigerator \\
\hline Enfermedad & Pelvis & Recogedor & Balcón & Illness & Pelvis & Dustpan & Balcony \\
\hline Ovarios & Desgarrador & Mesa & Archivo & Ovaries & Heartbreaking & Table & File \\
\hline Hormonas & Ginecólogo & Techo & Zafacón & Hormones & Gynecologist & Roof & Wastebasket \\
\hline Coágulos & Penetrante & Llave & Cepillo & Clots & Piercing & Key & Paint \\
\hline Ovulación & Punzante & Mirar & Pintura & Ovulation & Stabbing & Look & Path \\
\hline Cirugía & Sufrimiento & Puente & Sendero & Surgery & Suffering & Bridge & Dryer \\
\hline Cáncer & Vómito & Fregar & Secadora & Cancer & Vomiting & Wash & Printer \\
\hline Agonía & Preocupación & Zapato & Impresora & Agony & Worry & Shoe & Ball \\
\hline Llorar & Abdomen & Cartón & Bola & Cry & Abdomen & Carton & Brush \\
\hline Hospital & & Cocinar & & Hospital & & Cooking & \\
\hline
\end{tabular}

\section{Data analysis}

Text files were generated from the Super lab software and analyzed in Microsoft Excel (2010) and the Statistical Package for the Social Sciences (SPSS) software (version 17.0). Descriptive statistics including frequencies and percentages were used to calculate demographic variables. For analysis of MEST data, Repeated Measures ANOVA's were conducted for latency retention time (reaction times) in seconds (independent variable) at the two different phases of the menstrual cycle and level of menstrual pain (dependent variable). The level of pain was divided using reported pain during menses using the VAS scale (low 1-3, moderate 4-6, severe 7-10). Additionally, ANOVA were performed for the MEST Reaction times during the different phases of the menstrual cycle and reported levels of pain within each phase. Further, ANOVA analysis were conducted to assess how the level of pain affected depression and anxiety symptomatology categories (BDI-II and BAI) during the different phases of the menstrual cycle. In order to further explore the relationship between level of menstrual pain and depression and/or anxiety on MEST performance, stepwise multiple linear regression analysis were conducted taking into account alcohol use, smoking, days of menstrual cycle and days of menses as potential variables.

\section{Results}

The socio-demographic information for the 40 women who participated in the study is summarized in Table 2. The sample consisted of mostly single, highly educated females. The majority of participants reported a menstrual cycle with duration of 25 to 28 days $(52.5 \%)$ and all were in good health, without a diagnosis of any menstrual disorder (Table 2). Self-reported levels of menstrual pain varied among subjects, the majority $(55 \%)$ reporting moderate pain levels (4 to 7 on the VAS scale) (Table 2), which was experienced predominantly for one to three days (Table 2). 
Table 2 Participants menstrual cycle duration and reported level of pain

\begin{tabular}{|c|c|c|}
\hline Demographic/Physiological factor & $\mathbf{N}$ & $\%$ \\
\hline \multicolumn{3}{|l|}{ Status } \\
\hline Single & 26 & 65 \\
\hline Married & 11 & 27.5 \\
\hline Divorced & 3 & 7.5 \\
\hline \multicolumn{3}{|l|}{ Education } \\
\hline High School & 6 & 15 \\
\hline Associates Degree & 3 & 7.5 \\
\hline Bachelor's Degree & 25 & 62.5 \\
\hline Master's Degree & 5 & 12.5 \\
\hline \multicolumn{3}{|l|}{ Menstrual cycle duration (Days) } \\
\hline $25-28$ & 21 & 52.5 \\
\hline $29-32$ & 12 & 30 \\
\hline $33-36$ & 7 & 17.5 \\
\hline \multicolumn{3}{|l|}{ Menstrual pain (VAS)/Days menses } \\
\hline $\operatorname{Low}(1-3)$ & II & 27.5 \\
\hline Moderate(4-6) & 22 & 57.5 \\
\hline Severe(7-10) & 7 & 15 \\
\hline \multicolumn{3}{|l|}{ Days of menses } \\
\hline 3-Jan & 36 & 90 \\
\hline 6-Apr & 3 & 7.5 \\
\hline I0-Jul & 1 & 2.5 \\
\hline
\end{tabular}

VAS, visual analog scale of pain

To examine differences in MEST performance between the two testing periods, a Repeated Measures ANOVA analysis was conducted using cycle phase (follicular, premenstrual) as the within variable, and level of pain (Low 1-3, Moderate 4-6, Severe 7-10) as the between variable for the response time to MEST (reaction times). This analysis showed a trend towards a difference between phases $(\mathrm{F}=3.34$, $\mathrm{p}=0.08$; Figure $1 \mathrm{~A})$, but not level of pain $(\mathrm{F}=2.20, \mathrm{p}=0.13$; Figure 1). Examination of potential differences at each phase using Oneway ANOVAs showed no differences at the follicular phase $(\mathrm{F}=0.01$, $\mathrm{p}=0.98$; Figure $1 \mathrm{~A}$ ). However, at the premenstrual phase, the level of pain appeared to significantly affect the reaction times on the MEST $(\mathrm{F}=3.30, \mathrm{p}<0.05$; Figure 1). Further analysis with Tukey's post-hoc's suggested that women with severe pain levels responded slower to the pain-related words during the MEST only during the premenstrual phase compared to women who reported mild levels of pain $(p<0.05$; Figure 1A). There was also a trend towards a difference in reaction times between women with severe and moderate levels of menstrual related pain during the pre-menstrual phase of their cycle $(\mathrm{p}=0.08$; Figure 1A). Overall, these data suggest that women with severe pain during menstruation have reduced MEST reaction times only during the few days prior to menses, i.e., days before their expected onset of pain.

We next examined if depression or anxiety levels affected the MEST responses, along with the level of menstrual pain (Figure 1BC). A linear regression analysis was conducted revealing that BDI raw scores during the premenstrual phase and the level of pain reported contributed to slower reaction times in MEST performance during the premenstrual phase $(\mathrm{R}=0.49$, Table 3$)$. This suggests that depression symptoms may affect an individual's ability to respond to menstrual related negative words (Figure 1B). No effects were seen at the follicular phase or within any other factors.

To examine if risk behaviors had any effects on psychological measures linear regressions were conducted. There was no indication that alcohol or tobacco use had any effect on the MEST performance (Table 3). In addition, based on linear regression analysis no effect was seen on MEST performance regarding the menstrual cycle duration or number ofdays of menses (Table 3 ).

Table 3 Linear regressions of MEST performance during the premenstrual phase

\begin{tabular}{lll}
\hline Neuro cognitive measures & $\mathbf{T}$ & $\mathbf{P}$ \\
\hline Level of Menses Pain & 2.36 & $0.02 *$ \\
BDI Premenstrual & 2.45 & $0.02 *$ \\
BAl Premenstrual & 1.06 & 0.3 \\
Alcohol & -1.04 & 0.3 \\
Smoker & $0.7 \mid$ & 0.48 \\
Days of menstrual cycle & $-1.4 \mathrm{I}$ & 0.17 \\
Days of menses & 0.8 & 0.42 \\
\hline
\end{tabular}

$*_{p}<0.05$, statistically significant
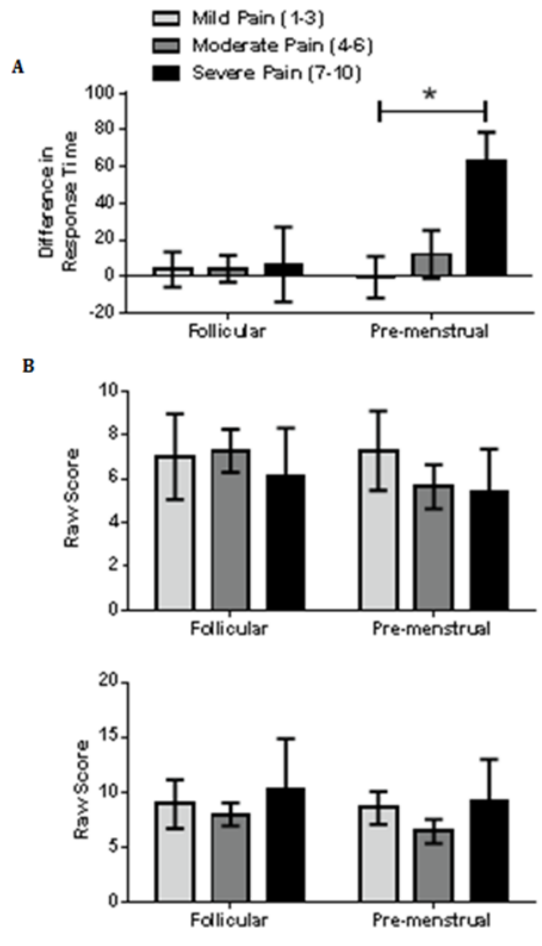

Figure I MEST performance, depression and anxiety levels at different phases of the menstrual cycle for women with difference levels of menstrual pain.

A) Women who reported severe pain showed significantly longer reaction times on the MEST $(p<0.05)$ compared to those with mild levels of pain.

B) No differences were seen in depression between menstrual phases or pain levels on the BDI-II.

C) No differences were seen in anxiety across menstrual phases or pain levels on the BAI. 


\section{Discussion}

The experience of menstrual pelvic pain is different among women; for some this monthly process is accompanied by severe pain that can negatively affect their bio psychosocial status and general wellbeing. The main aim of this study was to assess if healthy women would respond differently to pain-related stimuli during one phase of the menstrual cycle vs. another. Interestingly, our data show that this is true only for subjects who appear to have a negative experience associated with one of the menstrual cycle phases. We observed that women who reported high $(>7)$ levels of pain during their menses were more emotionally reactive to menstrual-related words, only when the MEST was administered during the premenstrual phase (late luteal phase). This effect was demonstrated by statistically significant slower reaction times on the MEST during this phase of the menstrual cycle. This finding suggests that for women with severe menstrual pain the burden of processing pain-related stimuli interferes with attention allocation to the more superficial task of labeling word color, resulting in longer MEST response latencies.

Nearly $80 \%$ of women experience some worsening of physiological and psychological symptoms a few days before the onset of menstruation, influencing well-being and behavior. ${ }^{32}$ Recent research has found additional evidence that links women's behavior with the different menstrual phases with the hormonal changes across these phase. ${ }^{33}$ Previous studies indicate that females react to emotionally negative stimuli differently in the follicular compared to the luteal phase. ${ }^{26,34}$ During the postmenstrual or follicular phase the levels of the ovarian steroid hormone progesterone are low, but pre-menstrually or during the luteal phase progesterone levels are higher. Many brain regions show phase-dependent progesterone receptor expression, such as the amygdala, brainstem, hippocampus, cerebellum and frontal cortex. Among those areas, the amygdala is a key region in emotional processing. ${ }^{34}$ Therefore, it is not surprising that females react emotionally to these normal hormone fluctuations. For some, the emotional impact can become problematic, as it may affect their cognition, attention and productivity at work or study.

Menstrual pelvic pain severity has been reported to be greatest during early follicular (menstruation) and premenstrual (late luteal phase) compared to late follicular and early luteal phases. ${ }^{8}$ Increased pain correlates with other indicators of emotional arousal during the luteal phase such as increased levels of stress hormones after a stressful task, higher amygdala activity when anticipating pain, and increased negative daily moods. ${ }^{34}$ Similar to our results Hoyer et al., ${ }^{26}$ found that women respond slower in the luteal phase to an EST consisting of emotionally charged words (e.g., happy or fearful) that are paired with faces. This supports our hypothesis that the MEST can be used to measure an individual's ability to pay attention and detect an increase in interference when menstrual-related emotional content is presented; moreover, this tool can be used to quantify differences in these responses in premenstrual (when severe pain is anticipated) vs. follicular phase.

To our knowledge, this is the first time a menstrual related EST has been configured to assess differences in menstrual pain perception during the two different phases of the menstrual cycle and to detect an increase in interference to pain-related stimuli, resulting in the configuration of the MEST. In our sample of relatively healthy young women, those with severe levels of menstrual pain presented significant changes in reaction to threatening words compared to neutral words only during the pre-menstrual phase of the menstrual cycle. This suggests that pain during the pre-menstrual phase is a factor that affects responses on the MEST. Thus, the MEST task might be utilized as a diagnostic tool to identify those women at risk of developing maladaptive emotional responses to their cyclic pain, which may in some women become pathologic (severe anticipatory stress that affect normal functioning of the individual). The MEST task could function as an intervention tool to be used as part of multidisciplinary management approach of severe menstrual related pain.

The significance of the results in the MEST with a non-clinical population shows the importance of understanding the menstrual cycle in healthy women. The observation that some of the women recruited in this study reported high levels of menstrual pain without a diagnosis of a gynecologic disorder indicate that there is a need for more education about what is considered normal menses and what may represent a pathology requiring of medical treatment. Independent of a diagnosis, when pain was severe these women showed signs of emotional interference when confronted with words that evoked the anticipation of pain. Anticipatory stress has been reported to occur in patients suffering from chronic disorders such as Irritable Bowel Syndrome (IBS), ${ }^{35}$ and linked to anxiety, disadvantageous decision making, ${ }^{36}$ and changes in neuroendocrine responses. ${ }^{37,38}$ The higher prevalence of chronic visceral pain in women has been related to gender differences in activation of the hippocampus, thalamus and cerebellum during tests that pair conditioned visual stimuli with painful rectal distensions..$^{39}$ Taken together, these data suggest that in women reactivation of old fearful memories is enhanced and may explain increased interference that we observed in the MEST. Interestingly, our data also shows that these fear responses are extinguished rapidly, as the MEST responses did not vary according to reported pain level during the proliferative phase of the menstrual cycle. It would also be interesting to ascertain if engaging in effective coping styles could help minimize the effects of anticipatory stress (effective copers vs. chronic ineffectiveness of coping).$^{40}$ More research is needed to understand the impact of anticipatory stress in women suffering from severe pain.

Considering that there is evidence of specific regions of the amygdala being involved in emotional interference task correlating to progesterone levels, neuroimaging of patients engaged in the MEST may be very interesting in future studies. ${ }^{14,16}$ We would hypothesize that the reaction time in the MEST premenstrually would correlate with amygdala reactivity in regions shown previously to be dependent on the phase of the menstrual cycle. ${ }^{12-15}$ Similarly, in response to fearful faces differences in amygdala reactivity would also be expected comparing premenstrual and follicular phases. ${ }^{41}$ This type of imaging studies would further aid in the understanding of brain regions involved in the emotion responses of women as a consequence of hormonal changes that occur during menstruation.

There are several limitations in this study. First the sample size can be considered to be small and not representative of the menstruating female population. Recruitment to this study was challenging as it required that participants agreed to come twice to the laboratory to conduct the MEST. The 40 women between the ages of 21 and 51 who completed the study were mostly single with a minimum academic level of high school, with no significant risk behaviors and healthy menstrual cycles, which was congruent with the desired inclusion criteria. We relied on participants' reported last menstrual period date (LMP) for planning the interventions; these were carefully planned by the investigator conducting the MEST. Thus, it is important that these findings are replicated using other methods. Attentional bias can be assessed in other ways, such as through the use of speeded probe detection tasks, priming techniques, visual search approaches and eye-movement methodologies. ${ }^{42}$ 
Studies with the MEST should be administered to women diagnosed with menstrual or gynecological disorders such as dysmenorrhea, endometriosis, amenorrhea, and PMDD among others. Such studies can provide further information and allow comparison with this non-clinical population regarding the negative effects of chronic menstrual pain and neuropsychological variables. Additional studies regarding the menstrual cycle and socio-demographics profile of healthy adolescents and young women ( $<21$ years old) should also be conducted to provide further understanding of the emotional impact of the menstrual cycle and pain perception among different ages and generations of women. Psycho-education on women's health and the menstrual cycle is highly recommended for all women at a very early age to equip women with the necessary tools to promote resilience and awareness to make informed decisions regarding their health.

Overall, our data suggest that emotional responses are deeply affected by the anticipation of menstrual pain and are associated with depression levels. This study also showed that MEST is an effective tool to obtain information about the emotional impact of menstrual pain via a non-invasive test. This test could help identify deficits in attentional processes caused by strong emotional stimuli elicited by menstrual-related stress. These results have implications for the development of pain management skills and psycho-education programs for women suffering from painful conditions such as endometriosis. Finally, our study suggest that the MEST is also a great tool to aid in the understanding of underlying processes and providing clinical assessment and therapeutic interventions to improve quality of life, allowing for the successful management of women's pain perceptions, increase resilience, and reduce the impact of menstrual pain in their physical and psychological well-being.

\section{Acknowledgements}

We acknowledge the support of Tirtsa Porrata-Doria and the Molecular Biology Core Lab and the Neuroscience Behavioral Core (Grant RR003050/8G12MD007579-27) at PHSU. Additionally, thanks to Rachel Dove for editing the manuscript AND TO THE INCREDIBLE COMMENTS OF JORGE PINZON!!!

\section{Conflict of interest}

Author declares that there is no conflict of interest.

\section{References}

1. Hawkins SM, Matzuk MM. The menstrual cycle: basic biology. Ann NY Acad Sci. 2008;1135:10-18.

2. Howard FM. Chronic pelvic pain. Obstet Gynecol. 2003;101(3):594-611.

3. Gunter J. Chronic pelvic pain: an integrated approach to diagnosis and treatment. Obstet Gynecol Surv. 2003;58(9):615-623.

4. Tettambel MA. Using integrative therapies to treat women with chronic pelvic pain. J Am Osteopath Assoc. 2007;107(10 Suppl 6):ES17-ES20.

5. Ayorinde AA, Macfarlane GJ, Saraswat L, et al. Chronic pelvic pain in women: an epidemiological perspective. Womens Health (Lond Engl). 2015;11(6):851-864.

6. Poleshuck EL, Bair MJ, Kroenke K, et al. Psychosocial stress and anxiety in musculoskeletal pain patients with and without depression. Gen Hosp Psychiatry. 2009;31(2):116-122.

7. Meltzer Brody S, Leserman J, Zolnoun D, et al. Trauma and posttraumatic stress disorder in women with chronic pelvic pain. Obstet Gynecol. 2007;109(4):902-908.

8. Tommaso M. Pain perception during menstrual cycle. Curr Pain Headache Rep. 2011;15(5):400-406.
9. Hamilton JA, Halbreich U. Special aspects of neuropsychiatric illness in women: with a focus on depression. Annu Rev Med. 1993;44(1):355-364.

10. Hamilton JA, Gallant S. Premenstrual syndromes: a health psychology critique of biomedically oriented research. Psychophysiology disorders: research and clinical applications. Washington: American Psychological Association; 1993. p. 383-438.

11. Berkley KJ, McAllister SL, Accius BE, et al. Endometriosis induced vaginal hyperalgesia in the rat: effect of estropause, ovariectomy, and estradiol replacement. Pain. 2007;132(Suppl 1):S150-S159.

12. Bannbers E, Gingnell M, Engman J, et al. The effect of premenstrual dysphoric disorder and menstrual cycle phase on brain activity during response inhibition. J Affect Disord. 2012;142(1-3):347-350.

13. Protopopescu X, Tuescher $\mathrm{O}$, Pan $\mathrm{H}$, et al. Toward a functional neuroanatomy of premenstrual dysphoric disorder. $J$ Affect Disord. 2008;108(1-2):87-94.

14. van Wingen GA, van Broekhoven F, Verkes RJ, et al. Progesterone selectively increases amygdala reactivity in women. Mol Psychiatr. 2008;13(3):325-333.

15. Andreano JM, Cahill L. Menstrual cycle modulation of medial temporal activity evoked by negative emotion. Neuroimage. 2010;53(4):1286-1293.

16. Han HJ, Lee K, Kim HT, et al. Distinctive amygdala sub regions involved in emotion modulated Stroop interference. Soc Cogn Affect Neurosci. 2014;9(5):689-698.

17. Stroop R. Studies of interference in serial verbal reactions. $J$ Experimental Psychol. 1935;6:643-662.

18. Larsen RJ, Mercer KA, Balota DA. Lexical characteristics of words used in emotional Stroop experiments. Emotion. 2006;6(1):62-72.

19. Wentura D, Rothermund K, Bak P. Automatic vigilance: the attentiongrabbing power of approach- and avoidance-related social information. J Pers Soc Psychol. 2000;78(6):1024-1037.

20. Christianson S. Handbook of memory and emotion: Research and theory. USA: University of Illinois Press. The American Journal of Psychology.1992. p. 476-479.

21. Reisberg D, Heuer F. Memory for emotional events. In: Reisberg D, Hertel P, editors. Memory and emotion. New York: Oxford University Press; 2004:3-41.

22. Kuyken W, Dalgleish T. Autobiographical memory and depression. Br J Clin Psychol. 1995;34(Pt 1):89-92.

23. Mathews A, Mackintosh B. A cognitive model of selective processing in anxiety. Cognitive Ther Res. 1998;22(6):539-560.

24. Lane RD, Sechrest L, Riedel R, et al. Pervasive emotion recognition deficit common to alexithymia and the repressive coping style. Psychosom Med. 2000;62(4):492-501.

25. Bailey HN, Paret L, Battista C, et al. Attachment anxiety and attentional control predict immediate and delayed emotional Stroop interference. Emotion. 2012;12(2):376-383.

26. Hoyer J, Burmann I, Kieseler ML, et al. Menstrual cycle phase modulates emotional conflict processing in women with and without premenstrual syndrome (PMS) a pilot study. PLoS One. 2013;8(4):e59780.

27. Cohen J. Statistical power analysis for the behavioral sciences. 2nd ed. NJ, USA: Hillsdale, Lawrence Erlbaum Associates, Inc; 1988.

28. Sommer B. Cognitive performance and the menstrual cycle. In: Richardson JTE, editor. Cognition and the menstrual cycle. New York: Springer Verlag; 1992. p. 38-66.

29. Beck A. BAI Spanish Translation. Texas: San Antonio, Pearson Education; 1993. 
30. Beck A. BDI-II Spanish Translation. Texas: San Antonio, Pearson Education; 1996.

31. Constans JI, McCloskey MS, Vasterling JJ, et al. Suppression of attentional bias in PTSD. J Abnorm Psychol. 2004;113(2):315-323.

32. Telek T, Gonda X, Lazary J, et al. The possible protective role of personality dimensions against premenstrual syndrome. Psychiatry Res. 2010;179(1):81-85.

33. Brown SG, Morrison LA, Calibuso MJ, et al. The menstrual cycle and sexual behavior: relationship to eating, exercise, sleep, and health patterns. Womens Health. 2008;48(4):429-444.

34. Sakaki M, Mather M. How reward and emotional stimuli induce different reactions across the menstrual cycle. Soc Personal Psychol Compass. 2012;6(1):1-17.

35. Berman SM, Naliboff BD, Suyenobu B, et al. Reduced brainstem inhibition during anticipated pelvic visceral pain correlates with enhanced brain response to the visceral stimulus in women with irritable bowel syndrome. J Neurosci. 2008;28(2):349-359.

36. Starcke K, Wolf OT, Markowitsch HJ, et al. Anticipatory stress influences decision making under explicit risk conditions. Behav Neurosci. 2008;122(6):1352-1360.
37. Elsenbruch S, Haag S, Lucas A, et al. Neuroendocrine and blood pressure responses to rectal distensions in individuals with high and low visceral pain sensitivity. Psychoneuroendocrinology. 2007;32(5):580-585.

38. Schulz P, Walker JP, Peyrin L, et al. Lower sex hormones in men during anticipatory stress. Neuroreport. 1996;7(18):3101-3104.

39. Benson S, Kattoor J, Kullmann JS, et al. Towards understanding sex differences in visceral pain: enhanced reactivation of classicallyconditioned fear in healthy women. Neurobiol Learn Mem. 2014;109:113-121.

40. Demyttenaere K, Nijs P, Evers Kiebooms G, et al. Coping, ineffectiveness of coping and the psychoendocrinological stress responses during in vitro fertilization. J Psychosom Res. 1991;35(2-3):231-243.

41. Derntl B, Windischberger C, Robinson S, et al. Facial emotion recognition and amygdala activation are associated with menstrual cycle phase. Psychoneuroendocrinology. 2008;33(8):1031-1040.

42. Mogg K, Millar N, Bradley BP. Biases in eye movements to threatening facial expressions in generalized anxiety disorder and depressive disorder. J Abnorm Psychol. 2000;109(4):695-704. 